\title{
Hybrid fibre optic sensor network for real-time high temperature performance monitoring of steel structures
}

\author{
Ying Huang* \\ Department of Civil Engineering, North Dakota State University, Fargo, ND 58018 United States
}

Email address:

ying.huang@ndsu.edu(Y. Huang)

\section{To cite this article:}

Ying Huang. Hybrid Fibre Optic Sensor Network for Real-time High Temperature Performance Monitoring of Steel Structures. International Journal of Civil Engineering. Vol. 1, No. 1, 2013, pp. 16-23. doi: 10.11648/j.ajce.20130101.13

\begin{abstract}
In and post a fire event, an accurate and real-time evaluation and monitoring of a structure's performance can assist firefighters for efficient survivor rescuing, which significantly improve the fire rescuing safety both for fire fighters and the trapped survivors. However, due to the lack of durable sensors, the structural performance of steel structures in fire conditions is challenging to be evaluated in real time, especially when the associated civil structures are in a large scale. In this paper, a fiber optic sensor network is developed and used to monitor the structural performance of the steel structures in high temperature environments. The fiber optic sensor network has the capacity of real-time large strain measurement up to $12 \%$ and temperature of up to $700^{\circ} \mathrm{C}$ simultaneously. The capability of large strain measurement up to $12 \%$ enables the sensor system to monitor the strain distribution of steel structures during fire events in real time. An one-story one-bay steel frame (A36 steel) is used as an example in this paper to perform the structural performance assessment of steel structures in high temperature using the developed sensor network. The simulated fire tests were performed using high temperature furnace through gradual temperature increase from room temperature to $800{ }^{\circ} \mathrm{C}$ at a rate of $10{ }^{\circ} \mathrm{C} / \mathrm{min}$. The evaluated fiber optic sensor network consists of two long period fiber grating (LPFG) temperature sensors, five movable extrinsic Fabry-Perot interferometric (EFPI) large strain sensors, and two hybrid EFPI/LPFG sensors, which were distributed along the steel frame inside and around the heating zone of the frame. Experimental results demonstrated that the developed sensor network effectively monitored the plastic hinge formation and failure mode of the steel frame, approving the feasibility of the sensor network for steel structure evaluation in high temperature environments.
\end{abstract}

Keywords: Fiber Optic Sensor, Large Strain Measurement, Structural Health Monitoring (SHM), High Temperature Performance, Steel Structure

\section{Introduction}

Fire has been the major hazard worldwide and it claimed a large number of lives and caused a significant loss of properties. The failure of the World Trade Center on Sep 11 2001 was an example of the fire induced catastrophic failure of a steel structure. Building collapse is one of main reasons for the life loss in a fire event. During fire events, buildings are strained significantly with permanent displacements in addition to extremely high temperature environments, especially for steel structures which have a significant materials softening in a high temperature environment. An accurate and real-time structural performance monitoring of the steel structures could significantly improve rescuing efficiency of firefighters in fire events. In addition, an accurate evaluation of steel structures' behavior during fires could provide designers valuable information for structural design improvements. Based on the actual real-time structural performance evaluations, updates of the fire design code may result in more applicable and reliable fire design and fire prevention approaches. However, due to the simultaneous large strain and high temperature, few traditional sensing techniques can be sustained and applied. Electrical resistance gauges (strain gauges)[1], the most commonly applied strain measurement method, normally can only sustain a temperature below $200{ }^{\circ} \mathrm{C}$ and strain level less than $2 \%$. Since optical fibers can stand high temperature, they have been extended to their applications in extremely harsh environments. These increasing research efforts on all-optical sensors' technology, combined with emerging and demanding applications, had demonstrated a promising technological platform characterized by unique sensitivity, compactness, reliability, electromagnetic immunity, capability for real-time sensing, and low cost[2], 
promoting them to a preferable solution for real-world applications.

Among all-optic fiber sensors, fiber optic grating[3], Fabry-Perot interferometric (EFPI)[4], and hybrid sensors had been intensively investigated for multiple parameter measurements, especially the hybrid fiber Bragg grating (FBG) sensors. For simultaneous strain and temperature measurement, multiple hybrid fiber optic sensors had been developed such as a hybrid EFPI/chirped FBG sensor[5] and a FBG/EFPI/LPFG sensor[6]. Nguyen et al.[7] also incorporated a FBG sensor into a Lyot fiber filter (LFF) by fusion splicing the FBG and a section of high birefringence fiber (PM fiber) for same purpose. However, regular FBG sensors have their own drawbacks of low temperature durability up to $300{ }^{\circ} \mathrm{C}$, which is not able to be applied in fire conditions up to $800^{\circ} \mathrm{C}$.

Searching for high temperature measurement on the other hand, long period fiber gratings (LPFGs) become the best candidates. Han et al.[8] reported that two sequent LPFGs with positive and negative temperature sensitivities could be applied for simultaneous strain and high temperature measurements. Zhao et al.[9] presented a new design for a simultaneous strain and temperature measurement using a HiBi-FLM concatenated with a temperature-insensitive long-period grating (LPG) written in a photonic crystal fiber (PCF). Rao et al.[10] reported a hybrid fiber-optic sensor consisting of a LPFG and a micro EFPI which can achieve a reasonable measurement of strains under high temperature. However, due to elasticity limitation of the optical fibers, all these high temperature sensors were reported to be in small strain dynamic range, which does not meet the requirement of large strain measurement in fire conditions.

To increase the strain measurement span and keep the high temperature capability of the fiber optic sensor, in 2012, our research group introduced a hybrid movable EFPI/LPFG sensor which could simultaneously measure high temperature and large strain[11, 12]. In this paper, based on the developed hybrid fiber optic sensor, a sensor network has been developed and evaluated for high temperature performance monitoring of steel structures.

\section{Hybrid Fiber Optic Sensor Network}

\subsection{Operational Principle}

A hybrid LPFG/EFPI sensor was designed and fabricated by combining one $\mathrm{CO}_{2}$ laser induced LPFG with one EFPI. With 100 times lower strain sensitivity than temperature sensitivity, the LPFG component of the hybrid sensor is regarded as a temperature sensing component in the hybrid LPFG/EFPI sensor[11]. On the other hand, packaged by a glass tube only, the hybrid sensor has the EFPI component whose temperature sensitivity depends upon the thermal coefficient of the optical fiber and the glass tube of $0.5 \times 10^{-6}$ strain $/{ }^{\circ} \mathrm{C}$, which is $50 \mu \varepsilon$ strain change for every $100{ }^{\circ} \mathrm{C}$. For temperature range of $800{ }^{\circ} \mathrm{C}$, a maximum $400 \mu \varepsilon$ of strain variance caused by temperature for the EFPI sensing component is expected. Compared to the $12 \%$ of strain dynamic range, the temperature effect only induces a strain variance less than $0.3 \%$. Thus, the cross effect of temperature on strain measurement has been neglected in this study and the EFPI component of the hybrid sensor worked as the strain sensing component $[11,12]$. Figure 1 shows the schematic of a hybrid fiber optic LPFG/EFPI sensor structure.

Light coming through the input fiber will transmit through the two end faces of the EFPI cavity. Since the distance between the LPFG and the EFPI endface was very short, typically less than $5 \mathrm{~cm}$, the reflected light from the near-end face of the EFPI will be reflected by the LPFG mirrors. Thus, the two branches of secondly reflected lights (by the LPFG mirrors) form an interferometer at the optical signal analyzer (OSA) output spectrum together with the spectrum of the LPFG. The spatial frequency of the interferometer is only a function of the EFPI cavity length and its refractive index change which will not affect the transmission signal of the LPFG itself as a sensor component.

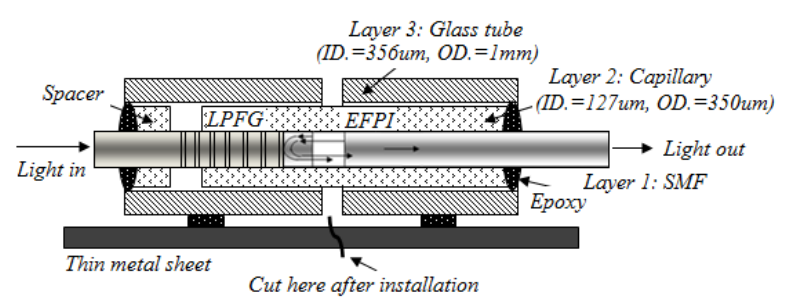

Figure 1. Structure and operational principle of a hybrid LPFG/EFPI sensor

\subsection{Signal Processing Algorithm}

Figure 2 (a) illustrates a typical optical spectrum of the hybrid LPFG/EFPI sensor and Figure 2(b) shows the Fast Fourier Transform (FFT) of the spectral interferogram. It can be seen from Figure 2(b) that the frequency components of the LPFG are low and with a cavity length of over $20 \mu \mathrm{m}$, the frequency component of the EFPI is higher than that of the LPFG. Thus, to multiplex the signals from the LPFG and EFPI components, a low-pass spectral Fourier transform filter was applied. The clean signal after filter can be applied to track the center wavelength of the LPFG sensor for temperature changes. On the other hand, the filtered signal can be used to monitor the cavity length of the EFPI based on the spectral frequency tracking method, resulting in strain information.

A hybrid LPFG/EFPI sensor protocol was fabricated and calibrated for simultaneous strain and temperature measurements. The hybrid sensor was installed on two steel channels of $2 \mathrm{~mm}$ apart, which were fixed on two computer controlled precise stages. The steel channels together with the hybrid sensor were further put into the high temperature furnace (supplied by Thermo Electron Corporation). Figures 2 (c, d) shows the multiplexed spectrum of the 
LPFG under various temperatures and its corresponding detected temperature changes in a Lab experiment. The hybrid LPFG/EFPI worked well up to $700{ }^{\circ} \mathrm{C}$.

For various strain levels, Figures 2 (e, f) show the multiplexed spectrum of the EFPI and their corresponding measured strains. As the gap distance changes from $20 \mu \mathrm{m}$ to $260 \mu \mathrm{m}$, the spatial frequency of the EFPI signal increases. By considering an EFPI gap change from $20 \mu \mathrm{m}$ to $260 \mu \mathrm{m}$, the gauge length and the initial gap distance of the EFPI part can be selected for a proper measurement of strain in various ranges. With the same initial gap distance of the EFPI (20 $\mu \mathrm{m}$ ), and the gauge length of the EFPI of $2 \mathrm{~mm}$, the strain can be measured up to $12 \%$.
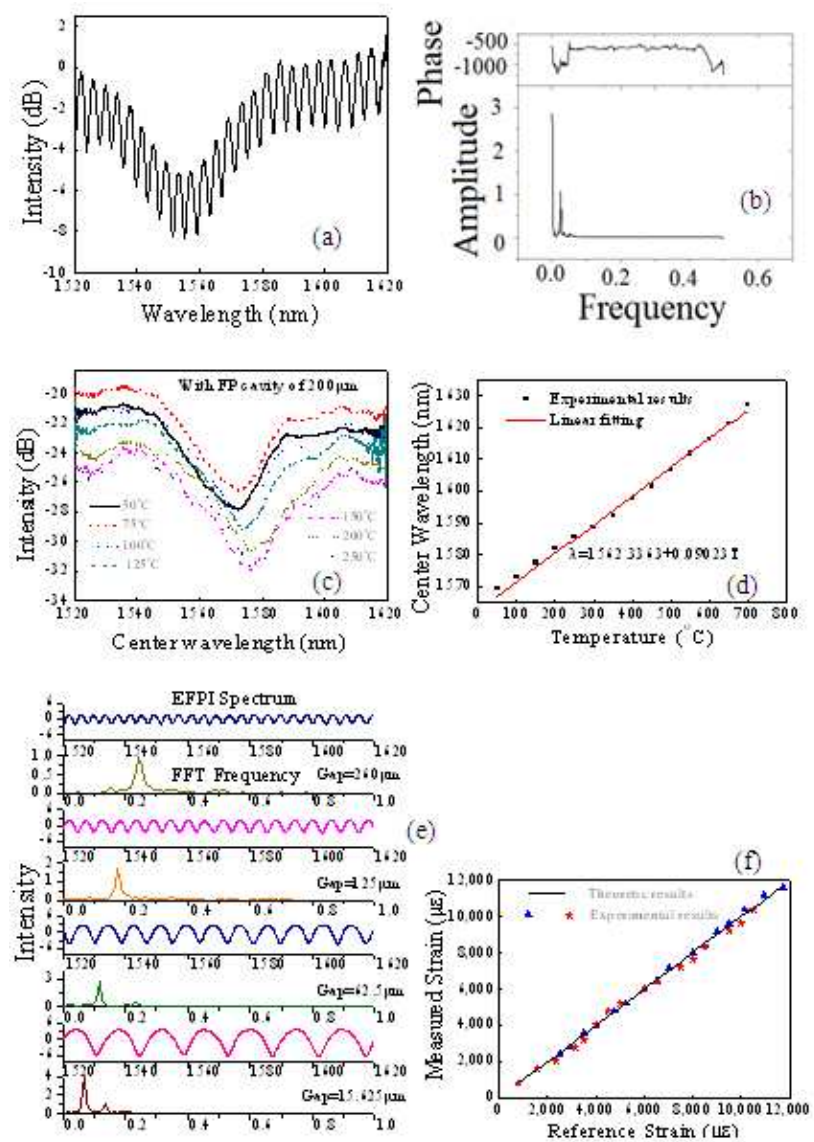

Figure 2. Signal processing of a hybrid LPFG/EFPI sensor: (a) typical sensor transmission signal spectrum, (b) FFT of sensor spectrum, (c) spectrum change vs temperature change, (d) wavelength change vs temperature, (e) spectrum change vs strain change, and (f) measured strain vs reference strain

\subsection{Multiplexing of Hybrid Fiber Optic Sensors}

In practical applications, to monitor the behavior of a large-scale civil engineering structure, a significant number of optical fiber sensors must be installed on the structure, which could amount the cost of a sensor network system to be non-negligible. Ideally, a multiplexed hybrid sensor system or network would be addressed by one input and one output fiber. Each sensor encodes the optical carrier with the information of sensed physical parameters, and the total optical output is conveyed to a detector and multiplexer that separate the encoded information relevant to each sensor into the appropriate number of channels for subsequent sensor demodulation and additional processing. However, in practice, more than one input and output fibers or a fiber sensor array may be required to address urgent civil engineering issues.

In this section, the development of a hybrid EFPI and LPFG sensor network for large strain and high temperature measurements. EFPI sensors have been widely applied for strain, chemical, and temperature measurements. However, they are difficult to multiplex due to limited cavity length. In the past three decades, more than six methods have been investigated for multiplexing fiber interferometric sensor arrays including frequency-division multiplexing (FDM) proposed[13], spatial-frequency multiplexing (SFDM) and wavelength-division multiplexing (WDM)[14 16], time-division multiplexing (TDM)[17 19], code-division multiplexing (CDM)[20], and their combinations. On the other hand, LPFG sensors are characterized by their high temperature endurance and high sensitivity to temperature changes. They can be inscribed along an optical fiber with a sufficient distance in between and multiplexed with the WDM methods. To maintain the workability of a large number of LPFGs in series, a cladding mode stripper between any two adjacent LPFGs must be developed[21].

In this study, for the developed hybrid sensor, we propose a combined FDW and WDM method for sensor multiplexing. Figure 3 shows the associated multiplexing network system for two hybrid LPFG/EFPI sensors. The two hybrid LPFG/EFPI sensors had been connected by two $1 \times 2$ couplers. Light coming out from the broadband source had been branched into $\mathrm{N}$ pieces at the first $1 \times 2$ coupler to each hybrid sensor and combined at the second $1 \times 2$ coupler to the optical signal analyzer (OSA). The OSA was further connected to a personal computer for the data processing to multiplex the signals for multiple LPFG and EFPI sensors. To distinguish the signals from an LPFG sensor and an EFPI sensor in the network system, the wavelength differences among LPFG sensors and the initial optical path differences among EFPI sensors were required.

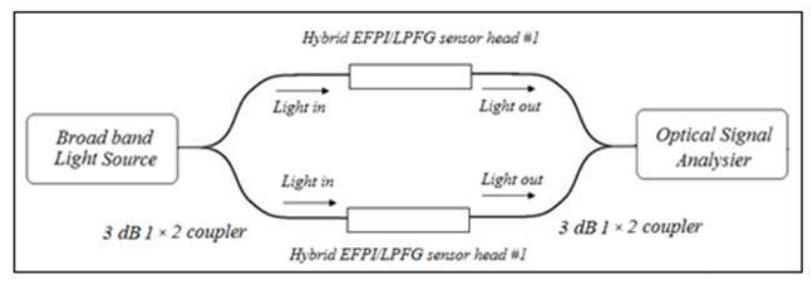

Figure 3. Multiplexing of the hybrid EFPI/LPFG sensors

To demonstrate the feasibility of the proposed hybrid sensor network above, two hybrid LPFG/EFPI sensors were fabricated and connected by two $1 \times 2$ couplers as illustrated in Figure 3, each having an LPFG component and an EFPI component. The individual spectrums of the two separate 
hybrid sensors are shown in Figure 4(a) while their combined spectrum is presented in Figure 4(b). The center wavelengths of the two LPFG components were set to differ by $10 \mathrm{~nm}$. The initial cavity lengths of the two EFPI components were $20 \mu \mathrm{m}$ and $60 \mu \mathrm{m}$ respectively. When the sensor network is installed on the same stage in previous tests and the stage generates a relative displacement of 40 $\mu \mathrm{m}$ on the optical fibers, the measurements from the sensor network are plotted in Figure 4(c). It can be seen from Figure 4(c) that the first peak represents a strain of $2.05 \%$ and the second peak gives $1.95 \%$. Their difference was within $5 \%$, providing consistent readings for the same strain imposed by moving the stage. With known spectral frequencies of both EFPI components, the spectrum of the network system can be filtered by a low-pass FFT filter. The LPFG spectrum after the FFT filter is presented in Figure 4(d). It can be seen that the LPFG components in the two hybrid sensors can be clearly distinguished and the sensor network is also capable of high temperature measurement. Therefore, the proposed quasi-distributed optical network system based on hybrid LPFG/EFPI sensors worked well for simultaneous large strain and high temperature measurements at multiple locations.
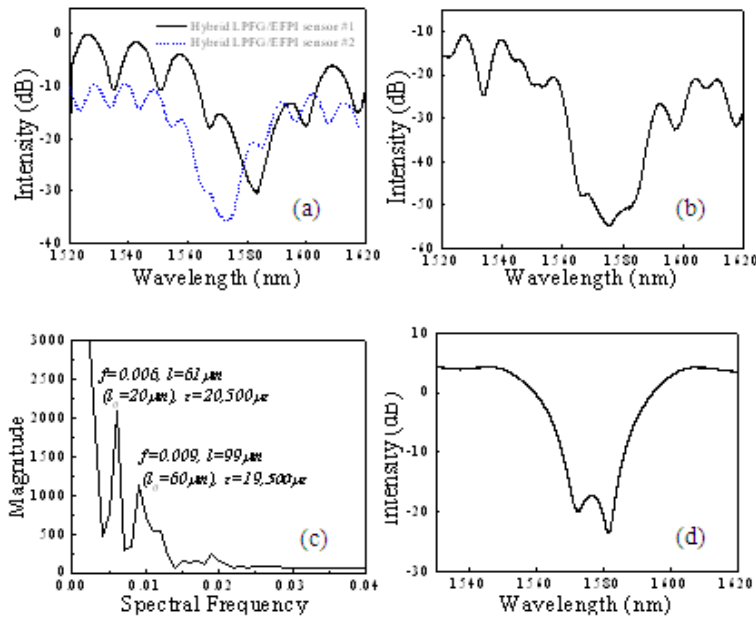

Figure 4. Experimental validation of the sensor multiplexing: (a) spectrum of two separate sensors, (b) spectrum of two multiplexed sensor, (c) multiplexed strains, and (d) multiplexed temperatures

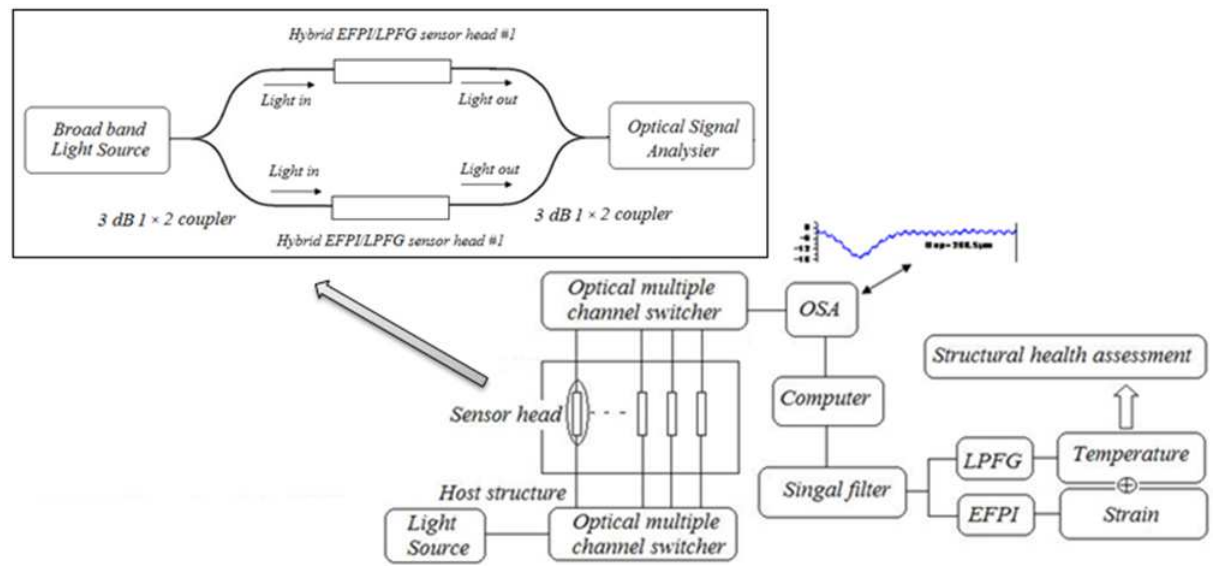

Figure 5. Integrated sensor network based on the multiplexed hybrid EFPI/LPFG sensors

\subsection{Network of Hybrid Fiber Optic Sensors}

To further increase the capability of the sensor system for large-scale structures, a sensor network based on the developed hybrid sensor is preferred by applying the proposed sensor multiplexing methodology. Figure 5 shows the proposed sensor network for structural health monitoring for large-scale civil infrastructure. In the network, $\mathrm{N}$ groups of multiplexed sensors are connected to two optical switchers with $\mathrm{N}$ channels to enable the network to measure $2 \times \mathrm{N}$ locations around the monitored structure. For example, with two 16-channel optic switchers, 32 key locations can be monitored on the critical structures for simultaneous large strain and high temperature.

\section{Experiments and Results}

\subsection{Experimental Setup}

To validate the developed fiber optic sensor network for structural monitoring and evaluation under high temperature environment such as fires in real time, in this study, a lab test was performed using a single-story, single-bay steel frame as illustrated in Figure 6(a). The steel frame was tested under combined dead and thermal loads as shown in Figure 6(b). The dead load was applied in the middle of the top beam using hydraulic loading cell. Corresponding to the increasing of furnace temperature, the frame was subjected to a vertical load of $20.46 \mathrm{kN}$ to 44.48 $\mathrm{kN}$ due to the expansion of the frame in high temperature environment. The thermal load was applied on the middle portion of the right column of the steel frame using a tube furnace (fabricated by Thermo Electron Corporation). The high temperature tube furnace has a dimension of $55.9 \times 137.2 \times 66 \mathrm{~cm}$, with $15.2 \mathrm{~cm}$ in inner diameter over a heating zone of $91.4 \mathrm{~cm}$ (with three heating zones shown in Figure 7: Zone 2 4). The temperature inside these three heating zone were controlled by the programmable controller for increasing to the same temperature 
simultaneously. To simulate the occurrence of high temperature environment caused by fire event, the temperature inside the three heating zones was gradually increased from room temperature to $800{ }^{\circ} \mathrm{C}$ with an increase rate $10^{\circ} \mathrm{C} / \mathrm{min}$. The temperature holds stable for 2 minutes whenever the furnace temperature reached $100{ }^{\circ} \mathrm{C}$, $200{ }^{\circ} \mathrm{C}, 300{ }^{\circ} \mathrm{C}, 400{ }^{\circ} \mathrm{C}, 500{ }^{\circ} \mathrm{C}, 600{ }^{\circ} \mathrm{C}, 700{ }^{\circ} \mathrm{C}, 750{ }^{\circ} \mathrm{C}$, and $800{ }^{\circ} \mathrm{C}$ for a stable measurement.
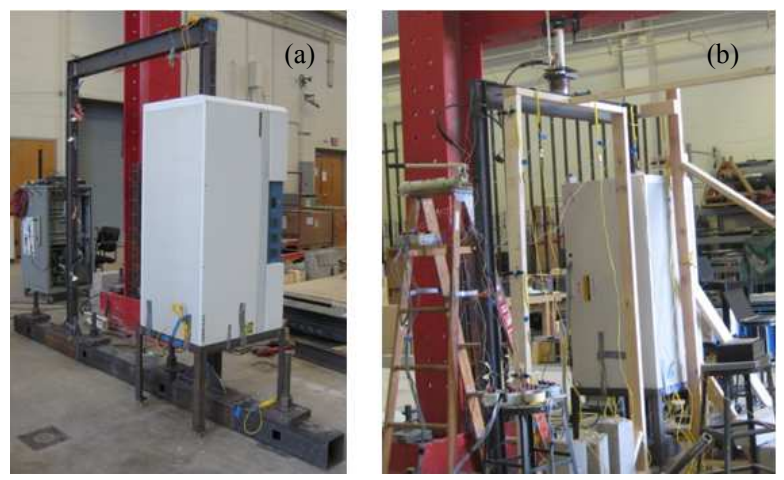

Figure 6. Experimental setup: (a) steel frame setup and (b) sensor implementation

The tested steel frame was made of hot-rolled S-shapes A36 mild steel. The center-to-center span of the horizontal beam, $\mathrm{S} 5 \times 10$, is $1.524 \mathrm{~m}$. The height of each column, $\mathrm{S} 3 \times 5.7$, to the center of the beam is $2.134 \mathrm{~m}$. The size of the columns was controlled by the dimension of the high temperature furnace. After additional spaces were considered for stiffeners and setup equipment, the length of the columns was selected to be $2.134 \mathrm{~m}$. The beam of the steel frame was selected to be 5.78 times as stiff as each column. The length of the beam was controlled by the $0.914 \mathrm{~m}$ anchor spacing on the strong floor in Structures Laboratory. To ensure a relatively rigid beam-column connection, three stiffeners were welded to each column. One $7.62 \times 15.24 \times 1.27 \mathrm{~cm}$ stiffener was placed on the top of the column, and the other two stiffeners, $3.56 \times 9.90 \times 1.27$ $\mathrm{cm}$, were placed on two sides of the column web to extend the bottom flange of the beam into the column flanges.

Both commercial and fiber optic sensor network were applied for a real-time structural assessment of the steel frame in high temperature environment. The commercial sensors were installed to validate the performance of the developed sensor network. Figure 7 shows locations of the sensors on the steel frame and the various temperature zones. The commercial sensing system consisted of ten K-type thermocouples (TM), five high temperature strain gauges (HSG), and fifteen regular strain gauges (SG). They were deployed outside the furnace. The fiber optical sensing system consisted of one fiber Bragg grating (FBG) temperature sensor, two LPFG temperature sensors[22], five movable EFPI large strain sensors[23], and two hybrid EFPI/LPFG sensors[12]. The FBG and one LPFG were placed immediately above the furnace on top of Zone 1 to monitor the temperature and strain at this transition area.
One LPFG temperature sensor was located at the base of left column to ensure a good estimate on the boundary condition. Three out of the five EFPI sensors (EFPI \#6, \#7, and \#2) were placed at the end of the heating zone, on the top of Zone 2 and $5(91.44 \mathrm{~cm}$ total height) and two (EFPI \#3 and \#4) were located at the third and two-third points which is on the top of Zone 4 and 3 as illustrated in Figure 7. One hybrid LPFG/EFPI sensor (EFPI \#1 and LPFG \#1) was placed at the bottom of the furnace and the other (EFPI \#5 and LPFG \#2) was at the 2/3 length from the bottom of the furnace (on the top of Zone 3). Ceramic adhesives that can endure high temperature up to $1100{ }^{\circ} \mathrm{C}$ were used to attach sensors on the inside surface of the column flanges.

\subsection{Experimental Results}

\subsubsection{Temperature Measurements}

Figure $8(\mathrm{a})$ plots the measured temperature by thermocouples as a function of time when the furnace temperature increased to $800{ }^{\circ} \mathrm{C}$. The temperature of the top beam and Column \#1 remained nearly unchanged since these members are away from the furnace by at least 30.48 $\mathrm{cm}$. The bottom of right column remained at room temperature because it was connected to a large area of the steel tube and then the reinforced concrete strong floor. This observation validated the room temperature assumption at structural boundaries. However, on the top of right column, temperature increased dramatically for more than $316^{\circ} \mathrm{C}$ as the furnace temperature rose to $800{ }^{\circ} \mathrm{C}$. Figure $8(\mathrm{c})$ shows the measured temperatures by various LPFG sensors. LPFG\#2 was placed inside the furnace. In addition, LPFG\#1, 3 , and 4 were respectively located near TM\#10, 9, and 1 . It can be observed from Figure 8(c) that the LPFG measurements are in good agreement with those by thermocouples, further validating the performance of optical fiber sensors up to the measurement range limit of thermocouples.

\subsubsection{Strain Measurements}

Figure 8(b) shows the measured strains by strain gauges as the furnace temperature increased to $800^{\circ} \mathrm{C}$. For the high temperature strain gauges, only HSG\#3 5 worked properly. Among the three functional HSG sensors, only HSG\#5 installed at the low end of the furnace seemed to give stable strain measurements prior to temperature compensation. Figure $8(\mathrm{~b})$ indicates that the steel frame structure outside the direct temperature loading zone remained elastic over the duration of tests. Figure 8(d) shows the measured strains by EFPI sensors. All the strains inside the furnace exceeded $0.2 \%$ when the furnace temperature reached $250{ }^{\circ} \mathrm{C}$, indicating the early initiation of yielding of the steel frame. The strain continued to rise as the temperature increased and the maximum strain obtained as presented in Figure 8(d) was slightly over $10 \%$ from EFPI\#6 since the EFPI sensor was designed for a maximum strain of $10 \%$ with an initial cavity of $50 \sim 60 \mu \mathrm{m}$. Thus, EFPI\#6 on the top of the furnace ran out of measurement range after the furnace temperature exceeded $600{ }^{\circ} \mathrm{C}$. Other EFPI sensors measuring a 
maximum strain of less than $10 \%$ continued to function until the end of frame tests.

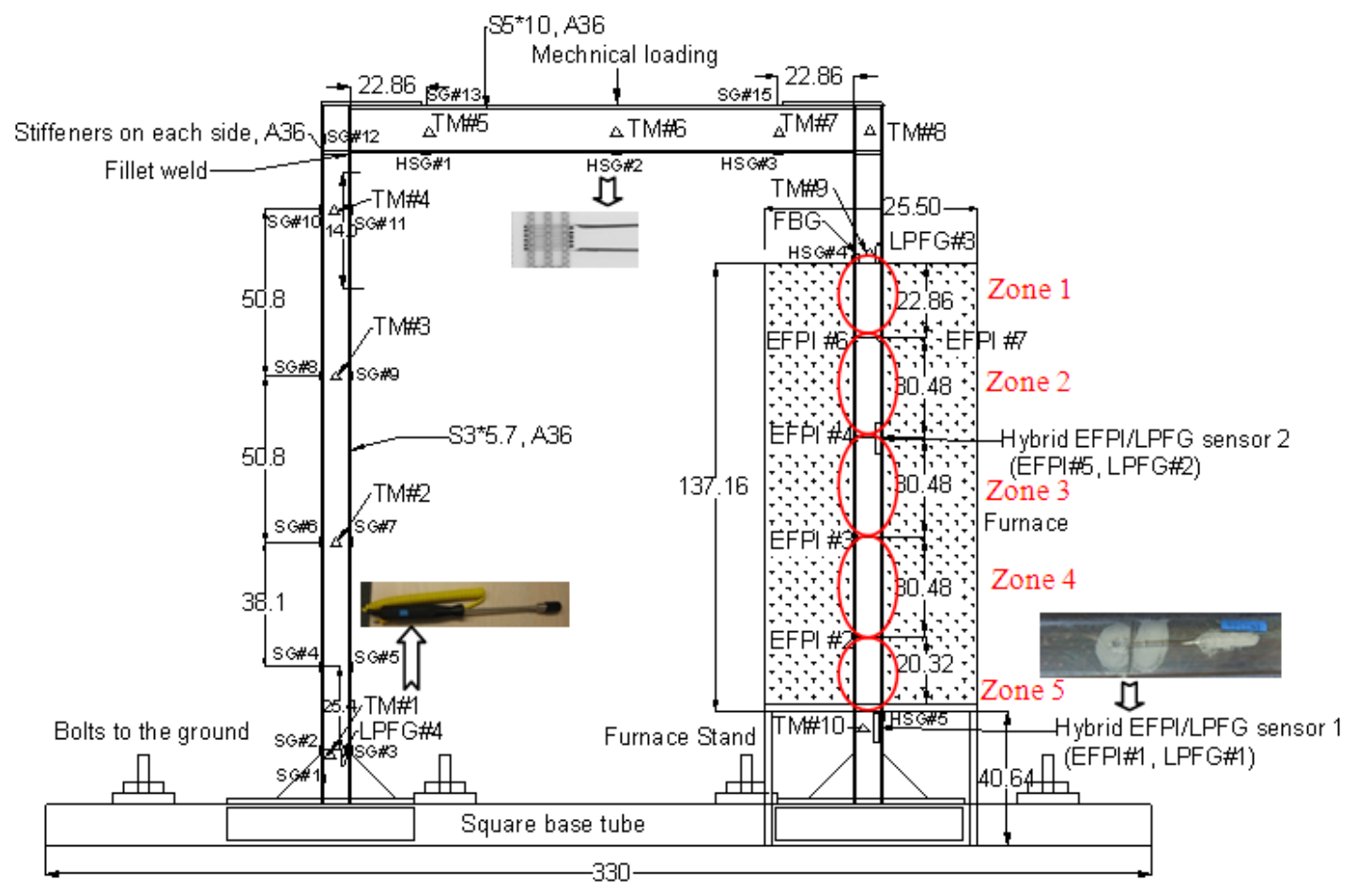

Figure 7. Sensor distributions on the validation test steel frame (all dimensions: $\mathrm{cm}$ )
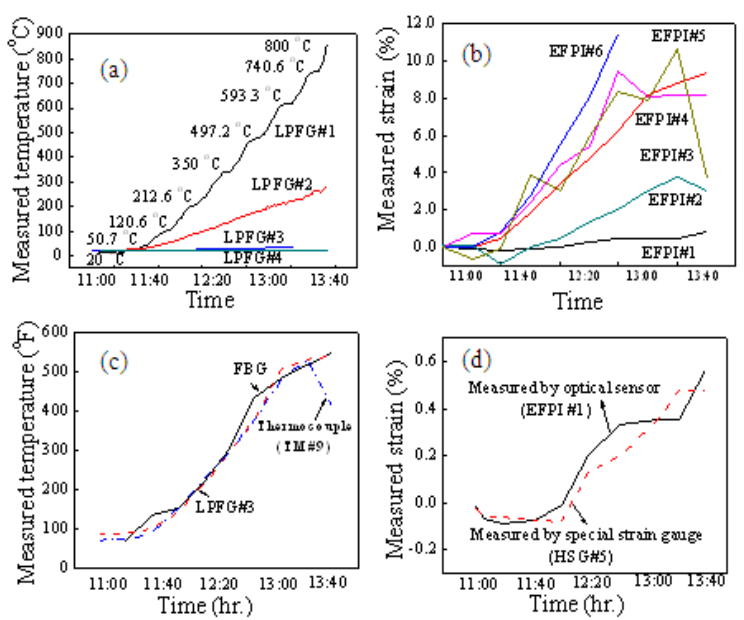

Figure 8. Experimental results: (a) measured temperatures, (b) measured strains, (c) measured temperature compared to commercial sensors, and (d) measured strain compared to commercial sensors

\section{Real-time Performance Evaluation of Steel Structure under High Temperature Conditions}

With the structural and environmental data monitored by the installed fiber optic sensor network in real time, the performance of the tested steel structure can then be evaluated. Real-time evaluation of the structural performance of the steel structure can be further used for decision making by the firefighters and the emergency management personnel.

\subsection{Temperature Influence on Steel Performance}

With concentrated high temperature (induced by local fire) on one particular region on a steel structure, in this study, in the middle portion of the right column of the single-store steel frame, the thermal gradient on the edges of these thermal loads are expected to be large. Figure 9(a) shows the thermal gradient of the steel frame on the edges of the thermal loading area (Zone 1 and Zone 5) derived in real time from measured temperature distribution of the hybrid sensors as indicated in Figure 8(a). Figure 9(a) showed that the bottom of the steel frame, Zone 1, has the largest temperature gradient compared to the top region of the thermal loading, Zone 5. This phenomenon is expected due to the gravity effect of the fire which always tries to bloom the hot air up and push the cool air down. The test results show that the thermal gradient on the bottom of the thermal loads at Zone 1 are two times larger than the top of the thermal loading area at Zone 5.

The high temperature gradient change at the bottom of the thermal loading area (Zone 5) induced a localized thermal stress at this particular location, which induced a dramatic plastic strain gradient in Zone 5 and Zone 4, in addition to the thermal induced elongation as shown in Figure 9(b). The high absolute temperature at top of the thermal loading area in combination of a big thermal gradient caused Zone 2 to have the biggest absolute plastic deformation but less strain 
gradient compared to Zone 4 due to the smaller temperature gradient in Zone 5. In temperature Zone 3, although a high absolute plastic deformation is observed, the strain gradient is low indicating a uniform temperature distribution along this zone.
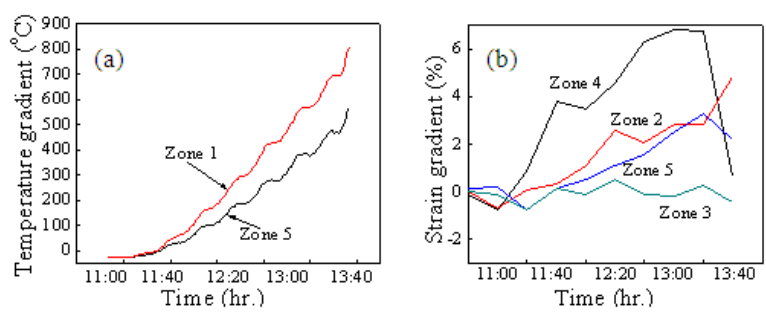

Figure 9. Real-time performance assessment of the steel frame: (a) monitored temperature gradient changes and (b) measured strain gradient changes

\subsection{Formation of Plastic Hinge}

Due to the high temperature gradient and at the same time great strain gradient, two plastic hinges were predicted from the real-time monitored data by the developed sensor network: one was located at Zone 4 and 5 which is at the bottom of the thermal loading area and the other was located at Zone 1 and 2 which is at the top of the thermal loading region. The bottom plastic hinge was initialized around the thermal loading temperature reaching $250{ }^{\circ} \mathrm{C}$ at Zone 4 and was extended to Zone 5 as thermal loads continued to rise. The top plastic hinge was initialized at Zone 2 when the thermal load reached $350{ }^{\circ} \mathrm{C}$ and continued to extend to Zone 1 as temperature increased. Figure 10 shows a photo of the tested frame after the performance assessment tests. Two plastic hinges were clearly observed in Figure 10, approving the real-time high-temperature performance assessment of the steel frame using the developed sensor network.

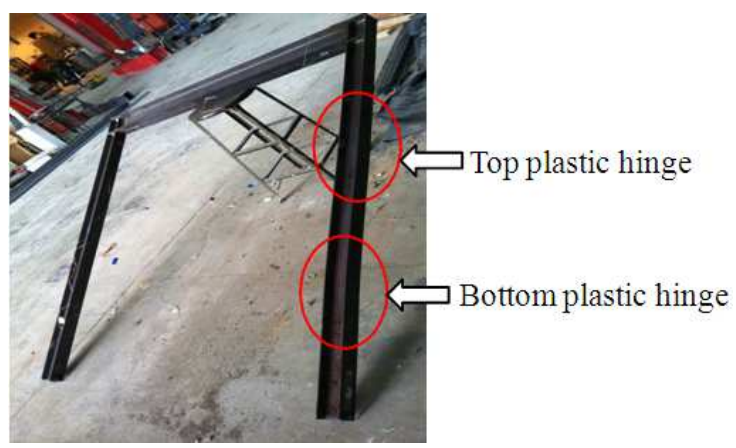

Figure 10. Formation of plastic hinges and failure of the steel frame

\subsection{Failure Mode of the Steel Frame with High Temperature Environments}

When the thermal loading reached $500{ }^{\circ} \mathrm{C}$ till the temperature went up to $650{ }^{\circ} \mathrm{C}$, Figure 8 (b) showed that the steel frame maintained at $8 \%$ strain inside the furnace and Figure 9(b) showed that the strain gradient at Zone 4 and Zone 2 stabilized. This indicated the initiation of the failure of the column. When the thermal loading reached between
$650{ }^{\circ} \mathrm{C}$ and $700{ }^{\circ} \mathrm{C}$, the measured strain gradient at Zone 4 and Zone 5 locations started to reduce, indicating the structural failure at bottom of the thermal loading region in a mode of compressive buckling. The measured strain gradient at Zone 2 on the other hand continued to increase, indicating a tension buckling at the top of the thermal loading region.

The strain gradient in Figure 9(b) also showed that both the bottom and top of the steel column's thermal loading region buckled in the week plane of the axis. The steel frame was cut down after tests and Figure 10 shows the visual inspection of steel frame's failure mode. It can be clearly seen from Figure 10 that the thermal loaded column of the steel frame was failed in week axis by buckling out of plane on the bottom and on the top, which agrees with the real-time monitoring assessment.

\section{Conclusions}

In this study, a fiber optic sensor network was developed to assess the performance of steel structures under high temperature environment in real time. Base on the systematic investigation, the following conclusions can be draw:

(1) The developed hybrid EFPI/LPFG sensor can simultaneously measure temperature up to $800{ }^{\circ} \mathrm{C}$ and strain up to $12 \%$;

(2) The developed hybrid sensor can be successfully multiplexed using a combined FDW and WDM method;

(3) The developed hybrid EFPI/LPFG sensor was successfully validated to monitor and evaluate the structural performance of an example steel frame by predicting its temperature influence on structural performance, the formation of the plastic hinges, and the failure locations and failure modes, approving its feasibility for high temperature performance evaluation.

The development of the proposed fiber optic sensor network enables the real-time performance assessment and evaluation of steel structures in fire conditions, which can be further applied for the assistance on fire hazard control and management, evaluation of fire design code, and structural designers for consideration of fire mitigations. The useful assessment information can also significantly help firefighters for efficient survivor rescuing, which would improve the fire rescuing safety both for fire fighters and the trapped survivors.

\section{Acknowledgements}

The author would like to thank Prof. Genda Chen and Prof. Hai Xiao from Missouri University Science and Technology for their facility supports in this study. 


\section{References}

[1] K. E. Easerling, "High temperature resistance strain gauges,” Brit. J. Appl. Phys., Vol. 14, No. 2, pp.79-84, 1963.

[2] V. G. M. Annamdas, "Review on developments in fiber optics sensors and applications," International Journal of Materials Engineering, vol. 1, No. 1, pp. 1-16, 2011.

[3] X. W. Shu, D. H. Zhao, L. Zhang, and I. Bennion, "Use of dual-grating sensors formed by different types of fiber Bragg gratings for simultaneous temperature and strain measurements," Applied Optics, vol. 43, no. 10, pp. 2006-2012, 2004.

[4] Y. J. Rao and D. A. Jackson, "Review article: Recent progress in fiber-optic low coherence interferometry," Meas. Sci. Technol., vol. 7, pp. 981-999, 1996.

[5] Y. J. Rao, S. F. Yuan, X. K. Zeng, D. K. Lian, Y. Zhu, Y. P. Wang, S. L. Huang, T. Y. Liu, G. F. Fernando, L. Zhang, and I. Bennion, "Simultaneous strain and temperature measurement of advanced 3-D braided composite materials using an improved EFPI/FBG system," Opt. Lasers Eng., vol. 38, pp. 557-566, 2002.

[6] X. K. Zeng, Y. J. Rao, Y. P. Wang, Z. L. Ran, and T. Zhu, "Transverse load, static strain, temperature and vibration measurement using a cascaded FBG/EFPI/LPFG sensor system," in: Technical Digest, OFS-15, Portland, USA, 2002, 199-202, 2002.

[7] L. V. Nguyen, D. Hwang, D. S. Moon, and Y. Chung, "Simultaneous measurement of temperature and strain using a Lyot fiber filter incorporated with a fiber Bragg grating in a linear configuration," Meas. Sci. Technol., vol. 20, pp. 034006-11, 2009.

[8] Y. G. Han and S. B. Lee, "Simultaneous measurement of temperature and strain using dual long-period fiber gratings with controlled temperature and strain sensitivities," Optics Express, vol. 11, no. 5, pp. 476-481, 2003.

[9] C. L. Zhao, J. R. Zhao, W. Jin, J. Ju, L. Cheng, and X. G. Huang, "Simultaneous strain and temperature measurement using a highly birefringence fiber loop mirror and a long-period grating written in a photonic crystal fiber," Optics Communications, vol. 282, pp. 4077-4080, 2009.

[10] Y. J. Rao, Z. L. Ran, X. Liao, and H. Y. Deng, "Hybrid LPFG/MEFPI sensor for simultaneous measurement of high-temperature and strain," Optics Express, vol. 15, no. 22, pp. 14936-14941, 2007.

[11] Y. Huang, G. Chen, H. Xiao, Y. N. Zhang, and Z. Zhou, "A quasi-distributed optical fiber sensor network for large strain and high-temperature measurements of structures," Proceedings of SPIE, Vol. 7983, Paper No. 798317, 2011.
[12] Y. Huang, X. Fang, W. J. Bevans, Z. Zhou, H. Xiao, and G. Chen, "Large-strain optical fiber sensing and real-time FEM updating of steel structures under high temperature effect", Smart Materials and Structure, vol. 22, pp. 015016, 2013.

[13] A. Dandridge, A. B. Tveten, A. D. Kersey, and A. M. Yurek, "Multiplexing of interferometric sensors using phase-generated carrier techniques," Electronics letters, vo. 23, no. 13, pp. 665-666, 1987.

[14] Y. J. Rao, J. Jiang, and C. X. Zhou, "Spatial-frequency-multiplexed fiber-optic Fizeau strain sensor system with optical amplification," Sens. Actuat. A, vol. 120, pp. 354-359, 2005.

[15] C. X. Zhou, Y. J. Rao, and J. Jiang, "A coarse-wavelength-division-multiplexed extrinsic fiber Fabry-Perot sensor system," Proc. SPIE, vol. 5634, paper no. 5634-32, 2004.

[16] Y. J. Rao, C. X. Zhou, and T. Zhu, "SFDM/CWDM of fiber-optic Fizeau strain sensors," IEEE Photon Technology Letter, vol. 17, pp. 1259-1261, 2005.

[17] A. D. Kersey, K. L. Dorsey, and A. Dandridge, "Demonstration of an eight-element time-division multiplexed interferometric fiber sensor array," Electronics Letters, vol. 24, no. 11, pp. 689-691, 1988.

[18] A. D. Kersey, "Demonstration of a hybrid time/wavelength division multiplexed interferometric fiber sensor array," Electronics letters, vol. 27, no. 7, pp. 554-555, 1992.

[19] D. G. Haigh and J. T. Taylor, "Time-division multiplexing of fiber optical interferometric sensors using a frequency modulated laser diode," Electronics letters, vol. 24, no. 1, pp. 54-55, 1988.

[20] A. D. Kersey, A. Dandridge, and M. A. Davis, "Code-division Multiplexed Interferometric Array With Phase Noise Reduction And Low Crosstalk, in Optical Fiber Sensors," in International Conferences on Optical Fiber Sensors, paper no.TH16, 1992.

[21] Y. G. Han, S. B. Lee, C. S. Kim, J. U. Kang, U. C. Paek, and Y. Chung, "Simultaneous measurement of temperature and strain using dual long-period fiber gratings with controlled temperature and strain sensitivities," Optics Express, vol. 11, no. 5, pp. 476-481, 2003.

[22] Y. Huang, Z. Zhou, Y. N. Zhang, G. Chen, and H. Xiao, “A temperature self-compensated LPFG sensor for large strain measurements at high temperature," IEEE Transactions on Instrumentation \& Measurement, vol. 59, no. 11, pp. 2997-3004, 2010.

[23] Y. Huang, T. Wei, Z. Zhou, Y. N. Zhang, G. Chen, and H. Xiao, "An extrinsic Fabry-Perot interferometer-based large strain sensor with high resolution", Meas. Sci. Technol., vol. 21, pp. 105308-16, 2010. 